\title{
HD5 and LL-37 Inhibit SARS-CoV and SARS-CoV-2 Binding to Human ACE2 by Molecular Simulation
}

\author{
Daixi Li ${ }^{1} \mathbb{D} \cdot$ Peiqin Chen ${ }^{1} \cdot$ Ting Shi $^{2} \cdot$ Aamir Mehmood $^{2} \cdot$ Jingfei Qiu $^{3}$
}

Received: 30 March 2021 / Revised: 30 June 2021 / Accepted: 2 July 2021 / Published online: 7 August 2021

(c) International Association of Scientists in the Interdisciplinary Areas 2021

\begin{abstract}
The coronavirus (COVID-19) pandemic is still spreading all over the world. As reported, angiotensin-converting enzyme-2 (ACE2) is a receptor of SARS-CoV-2 spike protein that initializes viral entry into host cells. Previously, the human defensin 5 (HD5) has been experimentally confirmed to be functional against the SARS-CoV-2. The present study proposes a human cathelicidin known as LL37 that strongly binds to the carboxypeptidase domain of human ACE2 compared to HD5. Therefore, LL37 bears a great potential to be tested as an anti-SARS-CoVD-2 peptide. We investigated the molecular interactions formed between the LL37 and ACE2 as well as HD5 and ACE2 tailed by their thermodynamic stability. The MM-PBSA and free energy landscape analysis outcomes confirmed its possible inhibitory effect against the SARS-CoV-2. The results obtained here could help propose a promising therapeutic strategy against the havoc caused by SARS-CoV-2 infections.
\end{abstract}

\section{Graphic abstract}
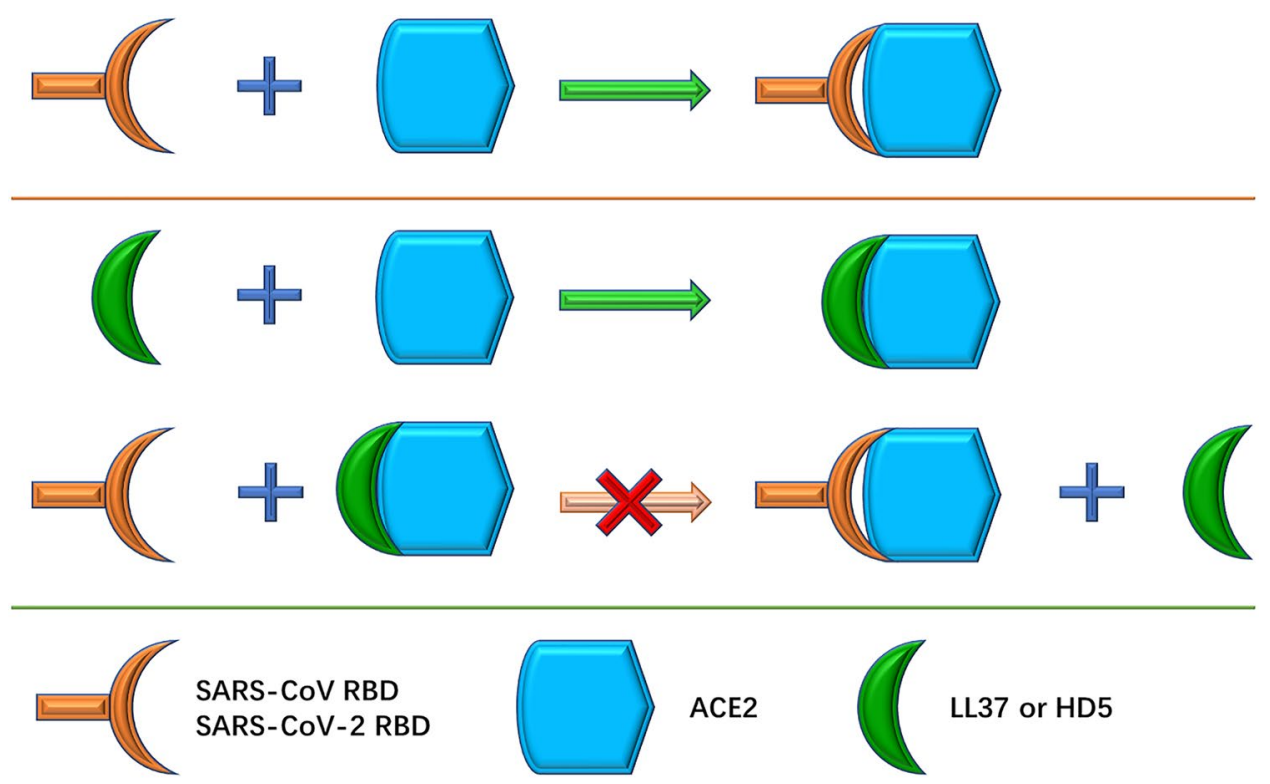

Keywords SARS-CoV-2 $\cdot$ SARS-CoV $\cdot$ Human ACE2 $\cdot$ MM-PBSA $\cdot$ Molecular simulation

\section{Introduction}

Daixi Li

dxli75@126.com

Extended author information available on the last page of the article
The coronavirus pneumonia pandemic (COVID-19) caused by the continuously mutating severe acute respiratory syndrome coronavirus 2 (SARS-CoV-2) [1] is still spreading 
rapidly all over the world, especially in the United States, Britain, India, and Vietnam. The clinical features of SARS$\mathrm{CoV}$-2-infected patients are similar to those infected by severe acute respiratory syndrome coronavirus (SARS$\mathrm{CoV}$ ) [2]. For instance, respiratory failure [3, 4], septicopyemia, cardiac failure [5, 6], hemorrhage [7], liver failure [8], hypertension [9], diabetes [9], and kidney failure [9] are considered as its leading causes of death.

The spike (S) glycoprotein is one of the main proteins in the SARS-CoV-2 [10] that is 1273 amino acids in length with 23 glycosylation sites linking 3-13 various six-membered sugar rings [11]. This $\mathrm{S}$ glycoprotein can be divided into $\mathrm{S} 1$ and $\mathrm{S} 2$ subunits after degradation by the protease. The $\mathrm{S} 1$ subunit contains a receptor-binding domain (RBD) devoted to the initial viral binding via recognizing host cell receptors, such as angiotensin-converting enzyme-2 (ACE2) because host cells expressing ACE2 is sensitive to SARS$\mathrm{CoV}-2$ [1]. The in vitro binding assay verified that ACE2 enabled a potent interaction with SARS-CoV-2 S protein with an affinity of $14.7 \mathrm{nM}$, which is 10 - to 20 -fold higher than that of the ACE2 binding SARS-CoV S protein [12]. On the other hand, studies have reported that the RBDs on SARS-CoV-2 and SARS-CoV bind with similar affinities to human ACE2 [11]. However, computational biologists at Pasteur Institute in Shanghai gave a different conclusion, stating that the interaction between $\mathrm{S}$ glycoprotein of SARS-CoV-2 and ACE2 was lower than that of the SARS virus based on theoretical simulation [13]. There is still no clear explanation to justify the conflict between the above statements.

Basic reports on the SARS-CoVID19 have maintained that the attachment of S glycoprotein with the carboxypeptidase domain of the ACED receptor in humans is crucial for the viral entry into the host cell. Thus, if any drug or peptide can avoid the complex formation of these two agents through binding with the carboxypeptidase domain with stronger affinity compared to the RBD will obstruct the viral entry into the host cell, expecting it to be a potentially powerful therapeutic strategy against the SARS-CoC-2 invasion. Therefore, it is expected to be an effective therapy against SARS-CoV-2 infection.

The innate immune system is a functional and physiological barrier against microbial infection, and antimicrobial peptides are essential effector molecules of this defense system. These antimicrobial peptides have broad-spectrum antimicrobial activities and many other biological functions. There are two kinds of antimicrobial peptides in the human body: defensin family peptide and cathelicidin family peptide. Although the defensin family peptide has many members, such as human defensins 5 (HD5) [14], cathelicidin has only one antimicrobial peptide, LL37 [15].

LL37 is the C-terminal part of the only human cathelicidin identified to date called human cationic antimicrobial protein (hCAP18), mainly expressed by neutrophils and epithelial cells as a putative growth factor for epithelial cells [16]. Additionally, the LL37 can be produced from human cells during mycobacterial infection, which exerts a microbicidal effect [17]. The hCAP18/LL37 as a multifunctional molecule mediates various host responses, including bactericidal action, chemotaxis, epithelial cell activation, angiogenesis, epithelial wound repair, and activation of chemokine secretion [18].

LL37 can kill bacteria and fungi directly [18] and demonstrate antiviral activity [19]. The in vitro analysis revealed that LL37 inhibits replication of human immunodeficiency virus -1 (HIV-1) in peripheral blood mononuclear cells [20], decreases vaccinia virus plaque formation and mRNA expression, and reduces the effect of infectious viruses such as herpes simplex virus -1 (HSV-1) and adenovirus-19 infection in A549 cells [21].

LL37 also has potent in vitro and in vivo antiviral activity against the influenza virus. Its treatment reduces the mortality rate, virus titer, and cytokine expression levels in mice's lungs challenged with a lethal influenza virus strain [22]. Besides, it effectively minimizes the number of RSVinfected HEp-2 cells and in plummeting the spread of RSV infection in HEp-2, as confirmed through in vitro analysis [23]. Therefore, LL37 plays an immunomodulatory role through chemotactic immune cells, regulating the secretion of inflammatory promoters/inhibitors, coordinating innate immunity and acquired immunity. Therefore, as a vital component of the innate immune system, only human cathelicidin LL37 plays an essential role in protecting humans against infectious diseases.

Depending on the action mechanism, recent studies have claimed that LL37, along with inactivating HSV-1, can be used as a therapeutic agent against SARS-CoV-2 [24]. Additionally, a new study found that LL37 attaches to the SARSCoV-2 spike protein [25] instead of just attaching itself to the membrane. It is also quite probable that the antimicrobial peptides LL-37 altered to decrease proteolysis and enhanced antiviral response will have an action against SARS-CoV2 [26].

Given the antiviral properties and biocompatibility of LL37, we studied the inhibitory effect of LL37 on SARSCoV-2 binding to human ACE2 through molecular simulations. Our results revealed that the LL37 free binding energy is stronger than HP5 towards ACE2, which we already verified theoretically and experimentally by analyzing the antiviral effect of HP5 on SARS-CoV-2 not long ago. Therefore, we hope that human cathelicidin will have an enhanced antiSARS-CoV-2 activity than the HP5. Fortunately, our above hypothesis has been confirmed by previous experiments [27]. Furthermore, since LL37 is an intrinsically disordered peptide, to demonstrate the binding flexibility and multitarget binding features of LL37, we have reported novel 
binding conformations with ACE2. Interestingly, both the binding conformations with ACE2 are stronger than RBDs.

\section{Theories and Methods}

\subsection{Data Retrieval and Model Preparation}

We accessed the RCSB protein databank to get the crystal structures of LL37 (PDB ID: 2K6O) [28] and the human ACE2 (PDB ID: 6ACG) [29]. The structure of LL37 is composed of a curved amphipathic helix-bend-helix motif spanning residues that ranged from 2 to 31 followed by a disordered C-terminal tail. The helical bend is located between the residues GLY14 and GLU16. The ACE2 crystallographic structure is composed of 597 residues ranging from SER 19 to ASP615 in the 6ACG's D chain [29].

The S glycoprotein of the SARS-CoV-ACE2 and SARSCoV-2-ACE2 complex was extracted from 6ACG and SARS-CoV-2 RBD/ACE2-B0AT1 complex [1] (PDB ID: $6 \mathrm{M} 17)$. For clarity, the RBDs on S glycoproteins of SARS$\mathrm{CoV}$ and SARS-CoV-2 were called RBD1 and RBD2 from here on. The biomolecular structures were visualized and rendered in PyMOL.

\subsection{Molecular Docking of LL37 on ACE2}

As discussed in our previous work on HP5 [30], we carried out the molecular docking between ACE2 and LL37 peptides on the ZDOCK server (http://zdock.umassmed. edu/) [31] using the default parameters. The dock site has 20 residues (SER19, GLN24, THR27, PHE28, ASP30, LYS31, HIS34, GLU35, GLU37, ASP38, TYR41, GLN42, LEU79, MET82, TYR83, ASN330, LEU333, LYS353, GLY354, ASP355) as shown in Fig. 1. After carefully considering all the docked conformations, we prioritized the best-docked orientations for molecular dynamics simulation to gauge their bonding stability.

\subsection{Molecular Dynamics Simulation}

To analyze the stability of docked conformations, we performed a molecular dynamics simulation of ACE2-RBD1 (PDB ID: 6ACG), ACE2-RBD2 (PDB ID: 6M17), ACE2HD5 (PDB ID: 6ACG and 1ZMP), and ACE2-LL37 (PDB ID: 6ACG and 2K6O) complexes. For this purpose, the GROMACS 2020.2 software package [32] was employed. The AMBER99SB-ildn forcefield [33] and the TIP3P water model were used for all the simulations, with a time step
Fig. 1 Dock sites of HP5 (a) and LL37 (b) on LDB of human ACE2: human ACE2 is highlighted in a pretty ribbon. Dock sites of RBD1 and RBD2 are identical and visualized in gray surfaces. The surface cloaked by HD5 is stained bright orange (a). And the surface cloaked by LL37 is highlighted in sky blue (b). The above visuals specify that the HD5 and LL37 have the potential to bind to the LBD of human ACE2
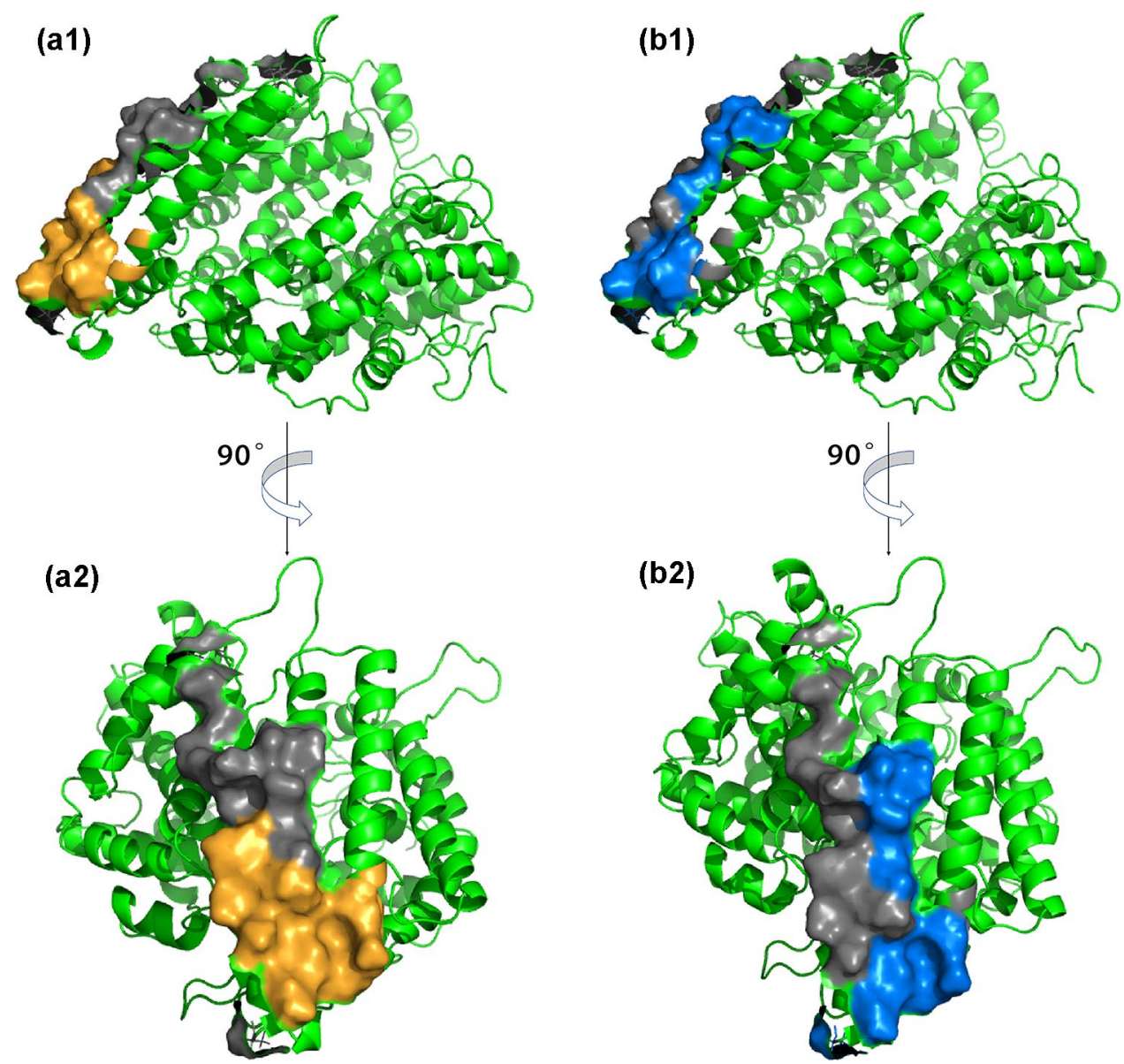
of $2 \mathrm{fs}$. Initially, 1000 steps of minimization were carried out. Next, for complete relaxation, 4 one ns pre-equilibration simulations with restrained coordinates of the heavy atoms, primary chain, backbone, and C-alpha were performed stepwise. Finally, a production simulation with an isothermalisobaric (NPT) ensemble at $1 \mathrm{~atm}$ and $300 \mathrm{~K}$ was conducted for a duration of $100 \mathrm{~ns}$. The last $5 \mathrm{~ns}$ of each trajectory were considered for the MM-PBSA calculation.

\subsection{Identification of Non-Covalent Interactions}

Non-covalent surface communications bear great importance. Therefore, to identify the non-covalent interactions at the interface of the human ACE2 complex with the RBD1, RBD2, HP5, and LL37, we used a freely accessible online tool known as PPCheck (http://caps.ncbs.res.in/ppcheck/ index.html) [34].

\subsection{Calculations of Thermodynamic Stability from MM-PBSA}

The molecular mechanics energies combined with the Poisson-Boltzmann surface area continuum solvation (MMPBSA) methods are popular approaches to estimate the free binding energy of small ligands and macro biomolecules
[35]. It is typically based on molecular dynamics simulations of the receptor- ligand complex and is intermediate in both accuracy and computational effort between empirical scoring and strict alchemical perturbation methods. MM-PBSA has consistently been proved to be a suitable approach to estimate binding free energy using a thermodynamics cycle. This is an attractive approach used widely to reproduce and rationalize experimental findings successfully, validating virtual screening and molecular docking results.

Therefore, the binding free energy was calculated by the MM-PBSA method with solvent accessible surface area (SASA) calculation using the g_mmpbsa tools [36] in GROMACS. In case of the binding free energy, we considered 5000 snapshots sampled after every $10 \mathrm{ps}$ from the overall $5 \mathrm{~ns}$ trajectory. From the RMSD, free energy landscape, and conformation cluster analysis (Figs. 2a and 3), it is observed that the change of binding entropy is small. The entropy effect is trivial because its estimation is a slow process and yields a small value.

\subsection{Dynamics Structural Stability Calculation}

Free energy landscape (FEL) derived from statistical results of dynamic structure can be used to discuss the structural stability of receptor-ligand complex formation [37]. We got the free (a)

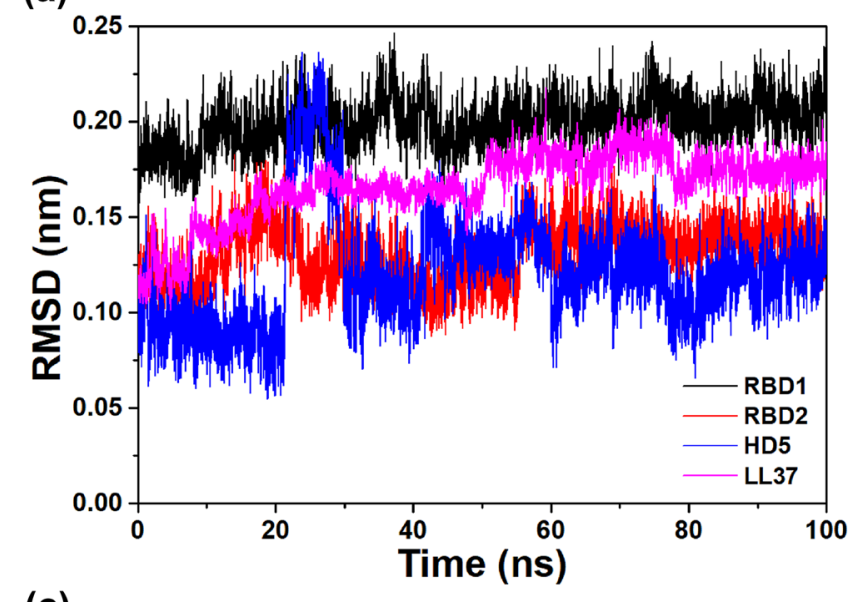

(c)

(b)

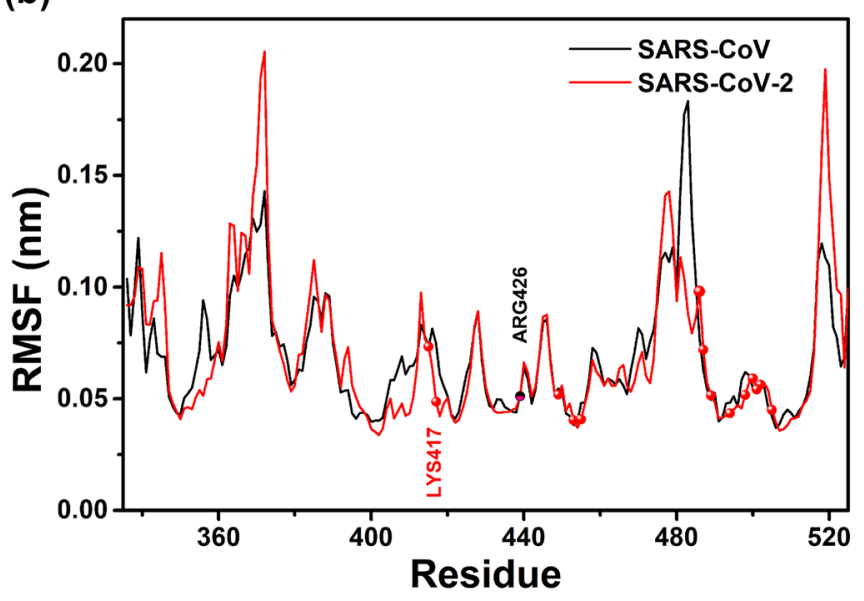

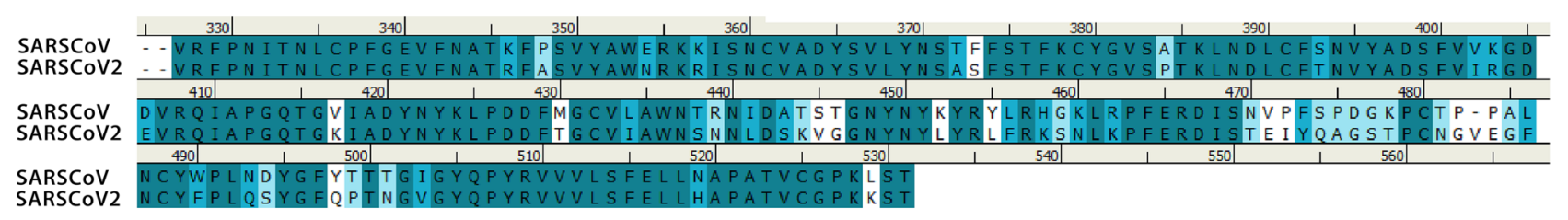

Fig. 2 Backbone RMSD of RBD1, RBD2, HD5, and LL37 in their corresponding complexes during simulation (a), residue RMSF of RBDs after binding to ACE2 (b), and their sequence alignment (c): four simulations reached their equilibrium at $60 \mathrm{~ns}$. The RMSF value indicated that the SARS-CoV-2 fitted with ACE2 as good as SARS-
CoV under normal conditions. Similar positions are correspondingly visualized in various navy and cyan fonts. The 14 RBD residues that are key for binding to ACE2 are highlighted in white. Sequence alignment resulted in sequence identity as $74.6 \%$ and similarity as $85.4 \%$ 

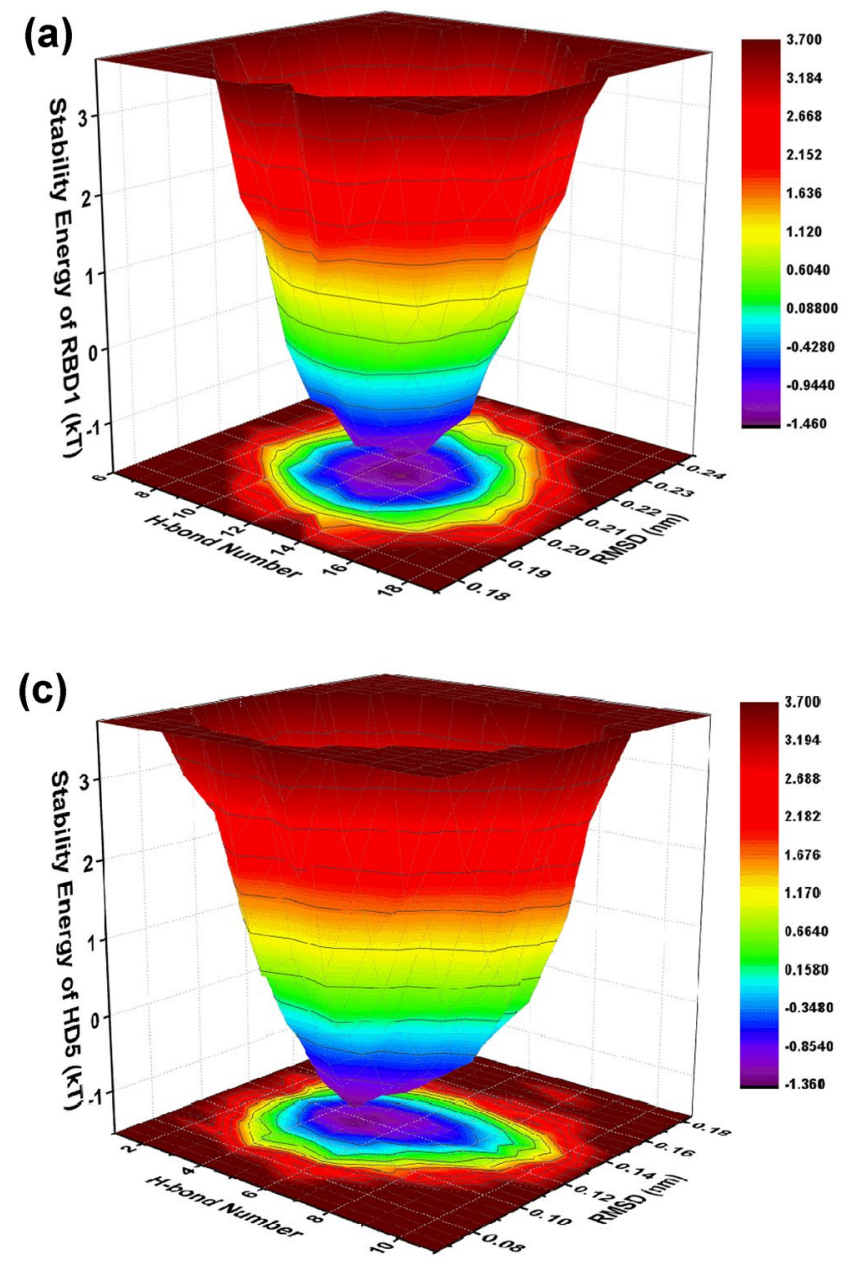

Fig. 3 Free energy landscapes of RBD1 (a) and RBD2 (b), HP5 (c), LL37 (d) binding to human ACE2, respectively: the $X$ axis is the number of H-bonds between human ACE2 and RBD1, RBD2, HP5, and LL37, respectively. The $Y$ axis presents the RMSD of the backbone atoms of RBD1, RBD2, HP5, and LL37. The $Y$ axis displays stabilizing energy built on 2D percent frequency statistics for the ratio

energy landscape of all the above docking complexes based on the 2D percent frequency statistics of the H-bond numbers and RMSD values derived from the last $40 \mathrm{~ns}$ trajectories after $60 \mathrm{~ns}$ equilibrated simulation. The H-bond numbers between the host (human ACE2) and each guest (RBD1 and RBD2, HP5, LL37) and the root mean square deviation (RMSD) of backbone atoms of each guest were analyzed from its corresponding $40 \mathrm{~ns}$ trajectories. We then used this data to investigate the structural dynamics of each guest after binding with the human ACE2.
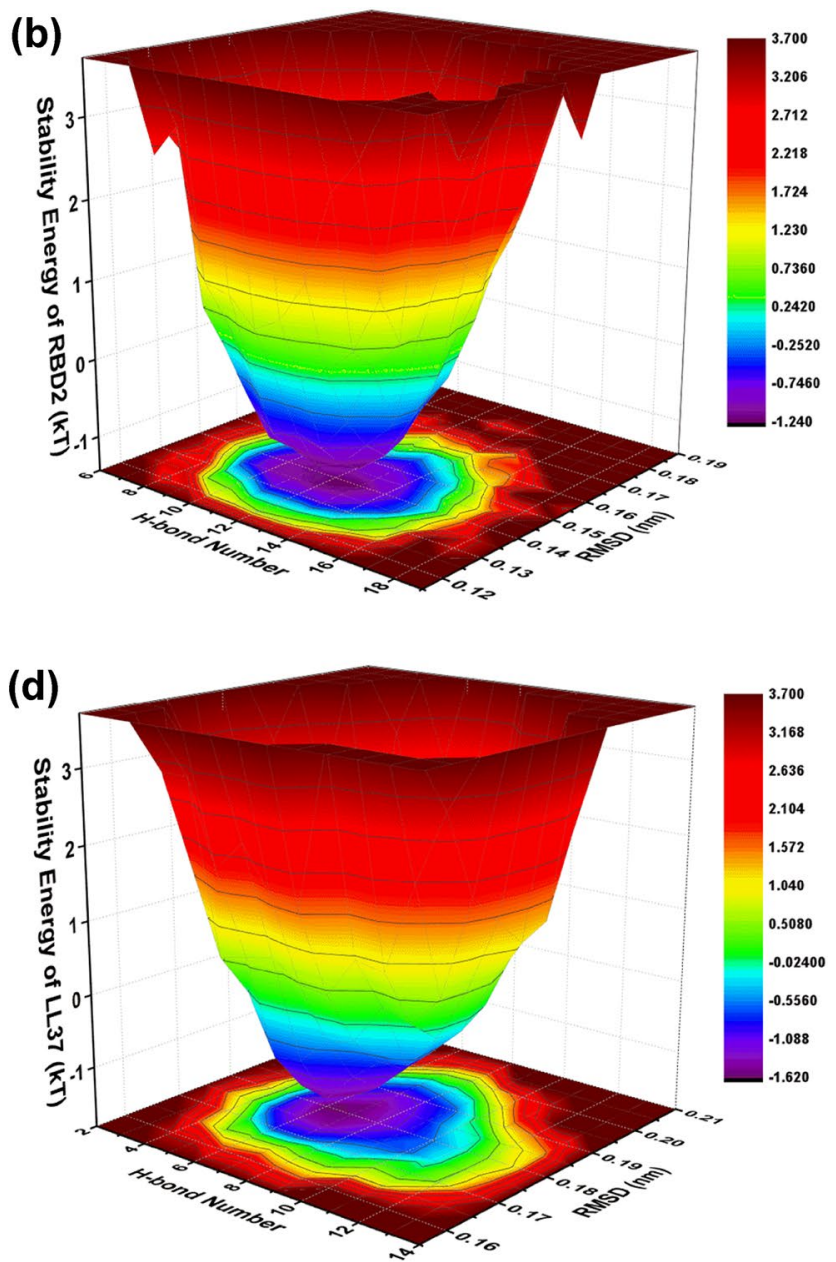

of H-bonds and RMSD values resulting from the last $40 \mathrm{~ns}$ trajectories. RBD1 ( $-1.46 \mathrm{kT})$ docked on the human ACE2 displays substantial dynamic stability than RBD2 $(-1.22 \mathrm{kT})$, though both are pretty similar. And the stabilizing energies show that LL37 (- 1.61 $\mathrm{kT}$ ) is also a potent inhibitor for hindering the infection of SARSCoV-2, same as HP5 (- $1.34 \mathrm{kT})$

\section{Results and Discussion}

\subsection{Key Interface Residues of Human ACE2 Hijacked by HP5 and LL37}

Previously, 14 key positions had been identified as crucial for the attachment of RBD1 to human ACE2 [38]. Among these 14,8 positions are strictly conserved in SARSCoV-2 S glycoprotein [39]. The remaining 6 positions 
are semi-conservatively substituted; namely ARG426ASN439, TYR442-LEU455, LEU472-PHE486, ASN479GLN493, TYR484-GLN498, and THR487-ASN501, where the formers belong to SARS-CoV and the latter belongs to SARS-CoV-2.

The conservation of many key interfacial residues could explain the similar binding affinities of RBD1 and RBD2 to human ACE2 [11]. Therefore, we used all the residues of human ACE2 within $5 \AA$ of RBD2 as a docking site of HP5 and LL37. The human ACE2 has 20 residues, among which 5 residues such as LYS31, GLU35, ASP38, MET82, LYS353 are vital for binding to RBD1 and RBD2 as previously reported [40] and confirmed from our simulation results. The docking sites are exposed in detail in Fig. 1.

PyMOL also calculated solvent-accessible interface areas (SAIA) of the above four equilibrated complex structures to investigate their structural stability. For SAIA, there was no significant difference between ACE2-RBD1 and ACE2RBD2. However, the interfacial area of ACE2-LL37 is much larger than that of ACE2-HP5 (Table S1).

For clarity, we analyzed the ACE2 complex structures with RBD1, RBD2, HP5, and LL37. According to the equilibrated structure of HP5 after 100 ns simulation, it engaged 11 interface residues of the human ACE2 (Tables S2-4). Among these residues, two are essential for binding to RDB: LYS31 and MET82. LYS31 established a strong H-bond (Table S2) and good electrostatic communication with GLU21 of HP5 (Table S3). And MET82 set 2 strong H-bonds with ARG 32 of HP5 simultaneously (Table S2).

Likewise, LL37 communicated ten interface residues of human ACE2 (Tables S5-8), among which three are crucial residues for binding to RDB: GLU35, ASP38, and MET82. GLU35 successfully established an H-bond (Table S5), two salt bridges (Table S6) with LYS25 of LL37, and two favorable electrostatic communications with LYS25 and ARG29 of LL37 (Table S7). ASP38 made a clear electrostatic linkage with ARG34 of LL37 (Table S7). Similarly, MET82 has an H-bond linkage (Table S5) with the LYS12 residue of LL37.

As shown in Fig. 2, it could be observed from RMSD results that RBD2 fitted better with ACE2 than RBD1. After comparing both the wildtype conformations, we observed that RBD1 has a higher RMSD value than RBD2, particularly in the case of mutated residues in the RBM in RBD2. This proves that under normal circumstances, the SARS-CoV-2 is more likely to combine with ACE2 than the SARS-CoV.

Once both of them reached their equilibrated state, the RBD2 interfacial residue gave a slightly smaller RMSF value than RBD1, confirming that the mutated residues at RBD2 have a more stable linkage with ACE2 than RBD1.

\subsection{Results of MM-PBSA Calculation}

The Gibbs binding free energy of LL37 with human ACE2 is the best of four guests (Table 1).

The MM-PBSA calculation showed that both the van der Waals and electrostatic interactions surged in the LL37ACE2-complex when compared to the rest of the complexes. For example, the LL37 van der Waals interactions were $73.38 \%$ and $15.35 \%$ higher than the HP5 and RBD2, respectively. At the same time, the electrostatic interaction of LL37 was $53.93 \%$ higher than that of HP5 and 100.29\% higher than that of RBD2. Therefore, LL37 should show better inhibition for the invasion of SARS-CoV-2 than HP5 since the binding Gibbs free energy of the former is more potent than that of the latter.

Interestingly, the interaction between RBD2 and ACE2 was lower than RBD1. This is consistent with the previously reported calculations [13]. Quite the reverse, the computational $\Delta \mathrm{G}$ did not confirm the experimental binding affinity by surface plasmon resonance (SPR) analysis [12]. Perhaps, there still exist gaps between theoretical calculation and the experiment side that need to be filled.

\subsection{The Dynamic Structural Stability of HP5 and LL37 with ACE2}

\subsubsection{The Free Energy Landscape and Dynamic-stable Energies}

We calculated the free energy landscape of the above complexes to compare the binding structural stability of each guest docking onto the human ACE2 (Fig. 3). The statistical results showed that all of the above complexes
Table 1 The binding energies calculated from MM-PBSA (unit: $\mathrm{kJ} / \mathrm{mol}$ )

\begin{tabular}{lllll}
\hline ACE2 & RBD1 & RBD2 & HP5/1zmp & LL37/2k6o \\
\hline $\mathrm{G}_{\text {Binding }}$ & $-1220.168 \pm 4.125$ & $-1148.534 \pm 4.106$ & $-1866.531 \pm 3.015$ & $-2769.300 \pm 6.152$ \\
$E_{\mathrm{vdw}}$ & $-359.513 \pm 1.202$ & $-371.021 \pm 0.959$ & $-246.843 \pm 1.079$ & $-427.965 \pm 1.253$ \\
$E_{\text {ele }}$ & $-1549.149 \pm 4.744$ & $-1491.26 \pm 2.688$ & $-1942.360 \pm 6.012$ & $-2989.910 \pm 5.430$ \\
$E_{\mathrm{p}}$ & $732.873 \pm 4.744$ & $756.807 \pm 4.818$ & $350.033 \pm 5.434$ & $697.337 \pm 5.519$ \\
$E_{\text {np }}$ & $-44.377 \pm 0.153$ & $-43.294 \pm 0.134$ & $-27.623 \pm 0.146$ & $-48.726 \pm 0.132$ \\
\hline
\end{tabular}

$G_{\text {Binding }}$ binding Gibbs free energy, $E_{v d w}$ van der Waal energy, $E_{\text {ele }}$ electrostatic energy, $E_{p}$ polar solvation energy, $E_{n p}$ SASA nonpolar solvation energy 
gave only one deep cluster, indicating that all of them were in their stable state after equilibration. Therefore, they should have minor changes in the conformational entropy prior to and post binding based on the thermodynamic cycle of MM-PBSA.

The ACE2-LL37 docked complex has the most stable dynamics structure since its relative stability energy is - $1.61 \mathrm{kT}$ while having $6 \mathrm{H}$-bonds and an RMSD value of $0.18 \mathrm{~nm}$. The ACE2-RBD1 relative stability energy is $-1.46 \mathrm{kT}$, thus ranking it the second most stable complex with $13 \mathrm{H}$-bonds and an RMSD value of $0.21 \mathrm{~nm}$. The third most stable docking complex is ACE2-HD5, with relative stability energy of $-1.34 \mathrm{kT}$, forming 5 $\mathrm{H}$-bonds and an RMSD value of $0.12 \mathrm{~nm}$. Finally, the remaining ACE2-RBD2 gained the relative stability energy of $-1.22 \mathrm{kT}$ and set $13 \mathrm{H}$-bonds with an RMSD value of 0.14 .

More importantly, RBD1 bound on the human ACE2 shows higher dynamic stability than RBD2 in terms of relative structural stability energy, although both are highly similar. This conclusion is consistent with the affinity results of the theoretical simulation [13]. That is to say, the SARS-CoV not only has a higher affinity but also binds more stable with ACE2 than SARS-CoV-2.

\subsubsection{Dynamics Structural Stability}

The host-guest conformation with the highest probability of occurrence extracted from the above free energy landscape analysis was the most dynamically stable. Typically, peptide binding on protein depends on their dynamic structural stability instead of a single crystal or NMR structural stability. Therefore, the dynamic structural stability of ACE2 complexes with HP5 and LL37 was analyzed, respectively (Fig. 4).

Since the binding of ACE2 with spike glycoprotein of SARS-CoV and SARS-CoV-2 had been reported many times previously, here, we only reported the summary result of their dynamic structural stability.

\subsubsection{Molecular Interactions Between ACE2} and HP5 According to the 2D percent frequency statistics for the H-bond numbers and RMSD values derived from the last $40 \mathrm{~ns}$ trajectories, we extracted the above four hostguest most dynamically stable conformations (Fig. 4).

The X-ray crystal diffraction has resolved the detailed structure of RBD2 bound with ACE2 at $2.45 \AA$ resolution [1], in which 12 residues of $\mathrm{N}$-terminal ACE2 constitute the ligand-binding domain (LBD). The HP5 peptide was docked on the N-terminal of the LBD of ACE2. The

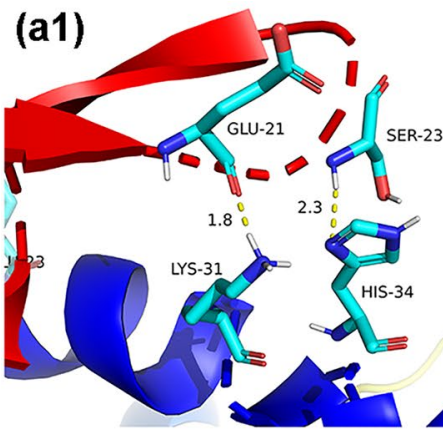

(a2)
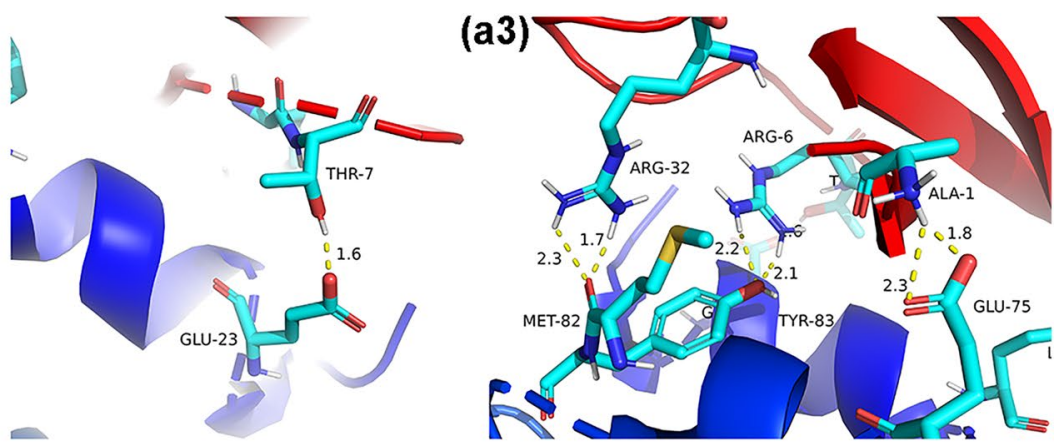

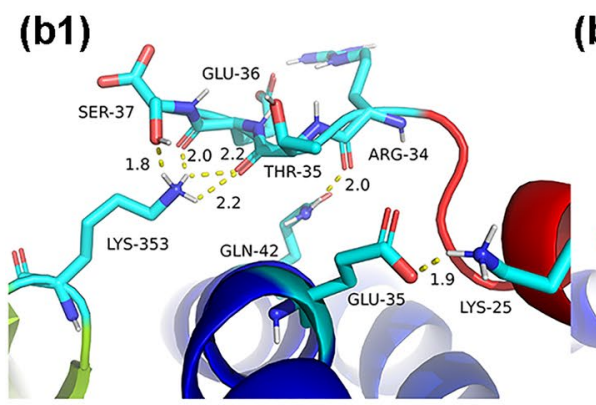

Fig. 4 H-bonding and salt bridge communication between ACE2 and HP5 (a), LL37 (b): the $\alpha$-helix (blue) is the LBD of ACE2, and the $\beta$-sheet (red) is the HP5 (a). The $\alpha$-helix in red was LL37 (b). There were $9 \mathrm{H}$-bonds in the most dynamically stable conformation of HP5. (b2) (b3)

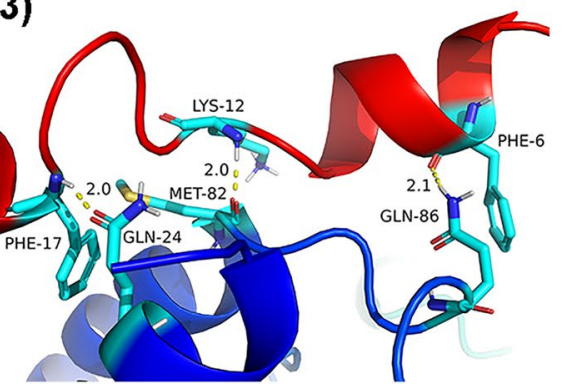

Totally, nine H-bonds and two salt bridges in the most stable conformation of LL37 can be observed. In (a3), the GLU75 and ALA1 form both the H-bond and salt bridges. In case of (b2), the GLU35 and LYS25 could establish both H-bond and salt bridge as well 
docked complex is so stable that its trajectory only gave a small RMSD value of about $0.12 \mathrm{~nm}$.

There were 53 interface residues in total, forming nine strong H-bonds between six pairs of residues (Table S2), five pairs of electrostatic interactions between HP5 and ACE2 (Table S3), and a hydrophobic center containing three pairs of hydrophobic interactions (Table S4).

There are $9 \mathrm{H}$-bond interactions between six pairs of residues on the interface of ACE2 and HP5 (Table S2). The HIS34, LYS31, GLU23, TYR83, MET82, and GLU75 of ACE2 established nine strong H-bond interactions with the SER23, GLU21, THR7, Arg6, ARG32, and ALA1 of HP5, respectively. Among them, the last three residues in each group formed two H-bonds simultaneously.

Also, four pairs of favorable electrostatic interactions and a pair of unfavorable electrostatic interactions between ACE2 and HP5 were spotted (Table S3). The GLU23, ASP30, LYS31, and HIS34 on ACE2 with ARG6, ARG25, GLU21, and GLU21 on HP5 formed four pairs of favorable electrostatic interactions with a distance of $8.58,9.15$, 8.23, and $8.47 \AA$, respectively. Next, the HIS34 on ACE2 with ARG25 on HP5 formed a pair of unfavorable electrostatic interactions with a distance of $9.41 \AA$ since the pair of residues have the same type of electric charge. The summary result of the above electrostatic interactions was favorable for binding.

There were three pairs of hydrophobic interactions between ACE2 and HP5 (Table S4). The sidechains of PHE28, LEU79, LEU79 of ACE2 and TYR4, ALA1, TYR4 of HP5 formed three pairs of hydrophobic interactions with a distance of $4.60,5.56$, and $5.86 \AA$, respectively. Thus, the sidechains of the above six residues on the interface compose a favorable hydrophobic center. The hydrophobic center significantly stabilized the complex of ACE2 and HP5 in the aqueous solution.

\subsubsection{Molecular Interactions Between ACE2} and LL37 For more in-depth insights into the ACE2 blocking of LL37, we carried out a molecular dynamics simulation, in which LL37 was docked onto the LBD of ACE2. After a 100-ns simulation, the complex conformation remained stable with minor fluctuations around $0.19 \mathrm{~nm}$ relative to the starting coordinates (Figs. 2a and 3d).

There are 82 interface residues in the equilibrated configuration, forming $9 \mathrm{H}$-bond interactions (Table S5), 2 salt bridges (Table S6), 9 electrostatic linkages (Table S7), and 7 hydrophobic contacts (Table S8).

There are nine $\mathrm{H}$-bond interactions on the interface of ACE2 and LL37 (Table S5). The GLN86, MET82, GLN24, GLU35, GLN42, and LYS353 of ACE2 formed nine strong H-bond interactions with the PHE6, LYS12, PHE17, LYS25, ARG34, THR35, SER37, and GLU36 of LL37, respectively. Among them, LYS353 of ACE2 links three residues THR35, SER37, and GLU36 of LL37 by four H-bonds.

There were two salt bridges between GLU35 of ACE2 and LYS25 of LL37, of which the measured distances were equal to 2.76 and $3.44 \AA$, respectively (Table S6).

Furthermore, we observed six favorable electrostatic communications between GLU35, ASP38, GLU87, and LYS353 of ACE2 and LYS25, ARG29, ARG34, ARG7, LYS10, and GLU36 of LL37 (Table S7), maintaining distances of 9.39, 8.93, 7.91, 7.42, 8.47, and $8.38 \AA$, respectively. Nevertheless, there are also three disapproving electrostatic linkages between ACE2 and LL37. The electrostatic interactions between LYS31, ASP38, GLU87 of ACE2 and LYS25, GLU36, and GLU11 of LL37 are unfavorable to attach with their distances' length equal to $9.65,6.40$, and $7.75 \AA$, respectively, since each electrostatic pair of residues poses the same charge. Opportunely, LYS25 was distant from LYS31. Besides, based on the final contribution of binding energy obtained from MM-PBSA analysis, GLU36, SER37, and GLU11 of LL-37 are the third most unfavorable residues to attach because the final contribution of GLU36, SER37, and GLU11 are $248.171 \pm 0.677 \mathrm{~kJ} /$ $\mathrm{mol}, 254.756 \pm 0.497 \mathrm{~kJ} / \mathrm{mol}$, and $258.965 \pm 0.769 \mathrm{~kJ} / \mathrm{mol}$, correspondingly. Therefore, as shown in the previous report [26], the GLU36, SER37, and GLU11 mutations in LL-37 could further enhance their binding.

There are two hydrophobic interactive cores on their interface (Table S8). One was LEU39, ALA71, PHE72 in ACE2 and LEU28, LEU31, and VAL32 of LL37. Another was PHE28, LEU79, and TYR83 of ACE2 and ILE13, PHE17, and ILE24 of LL37.

Finally, for comparison purposes, the final summary results are listed in Table 2.

3.3.2.3 Salt Bridge Salt bridge is a stronger intermolecular interaction than the H-bond. And the distance distribution between anion and cation pair is the key factor in measuring the salt bridge's strength. It is clear that both H-bond and salt bridge can be formed between the same pair of interfacial amino acids, which are the strongest interfacial interactions. Interestingly, there was a pair of interfacial amino acids that could form both $\mathrm{H}$-bonds and salt bridges in the above four host-guest complexes (Fig. 5).

In the ACE2-RBD1 complex, GLU329 (OE1 and OE2) of ACE2 and ARG426 (NH1 and NH2) of RBD1 could form two $\mathrm{H}$-bonds and four salt bridges at the same time. The percentage of their distance distribution in the salt bridge zone $(<0.4 \mathrm{~nm})$ was up to $97.98 \%$. And the percentage in the H-bond zone $(<0.35 \mathrm{~nm})$ was up to $88.62 \%$. The interaction was typically a stronger mixing interaction than the $\mathrm{H}$-bond plus salt bridges. The residue ARG426 might be crucial for the person--person transmission of SARS-CoV, but it is quite challenging to examine it in the crystal structure or 
Table 2 Interactions and total stabilizing energy in the most dynamic-stable conformation

\begin{tabular}{lllll}
\hline Items & SARS & SARS-CoV-2 & HP5 & LL37 \\
\hline H-bond number & $12(19)$ & $12(19)$ & $9(13)$ & $9(15)$ \\
Salt bridges & 4 & 2 & 1 & 2 \\
Electrostatic interactions & $5(1)$ & $3(1)$ & $5(4)$ & $9(6)$ \\
Hydrophobic interactions & 1 & 1 & 3 & 7 \\
van der Waals pairs & 8344 & 8629 & 6681 & 10,365 \\
Interface residues & 87 & 93 & 53 & 82 \\
H-bond energy (kJ/mol) & -75.20 & -80.31 & -64.10 & -69.87 \\
Electrostatic energy (kJ/mol) & 20.86 & -8.90 & -17.16 & -25.35 \\
VdW energy (kJ/mol) & -243.42 & -238.42 & -182.81 & -252.36 \\
Stabilizing energy $(\mathrm{kJ} / \mathrm{mol})$ & -297.76 & -327.63 & -264.07 & -347.58 \\
\hline
\end{tabular}

PPCheck server [34] only processes a single conformation. Therefore, no error was encountered in the above data. The number of $\mathrm{H}$-bonds enclosed in brackets means the highest $\mathrm{H}$-bond number that could be formed. Similarly, the electrostatic interactions in brackets suggest the amount of unfavorable electrostatic linkages (a)

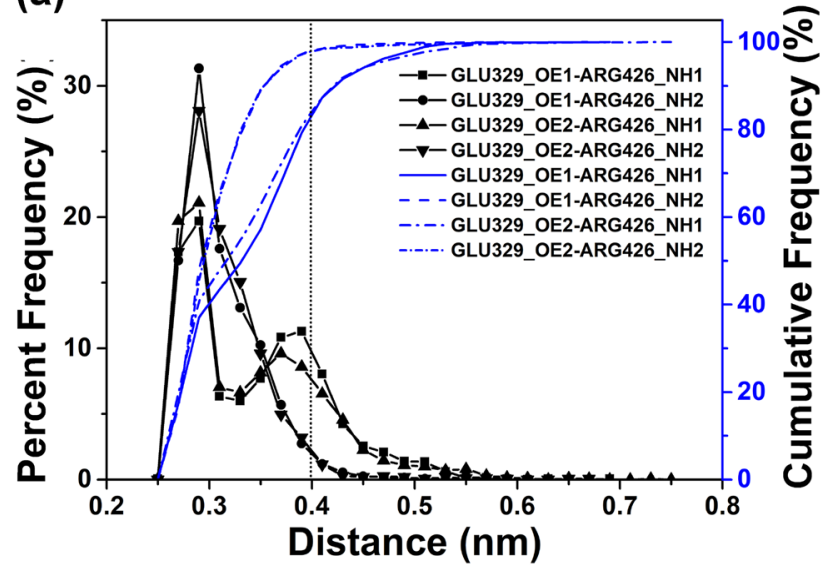

(c)

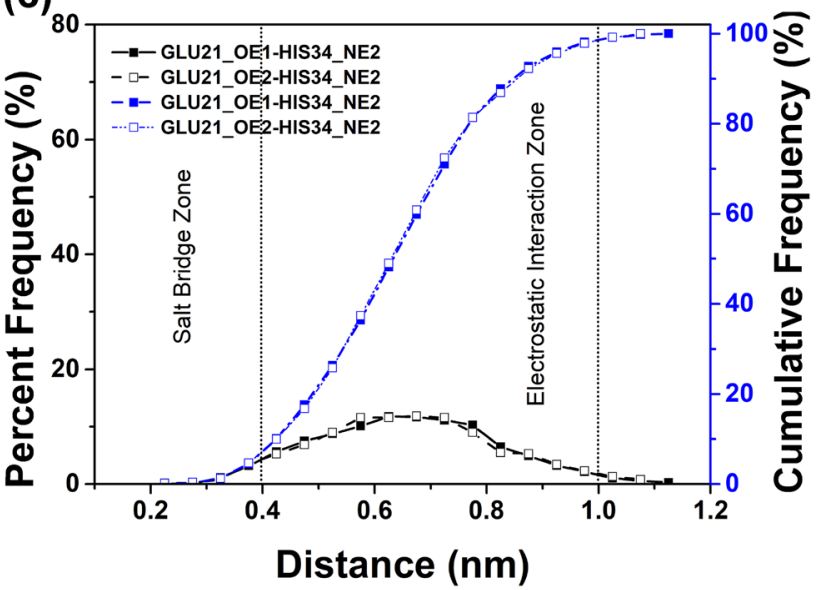

Fig. 5 The curves of the distance distribution of charge pair: a GLU329 (OE1 and OE2) of ACE2 and ARG426 (NH1 and NH2) of RBD1; b ASP30 (OD1 and OD2) of ACE2 and LYS417 (NZ1) of RBD2; c HIS34 (NE2) and GLU21 (OE1 and OE2) in ACE2-HP5 (b)

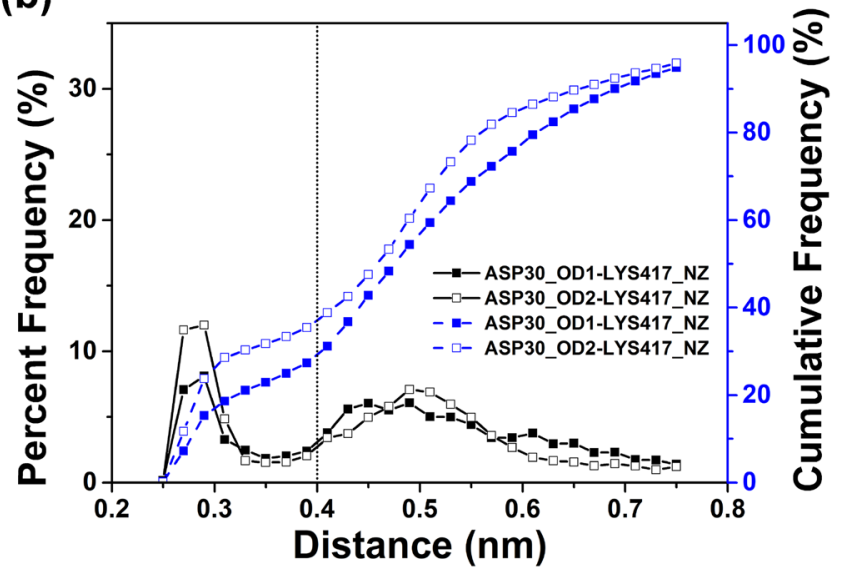

(d)

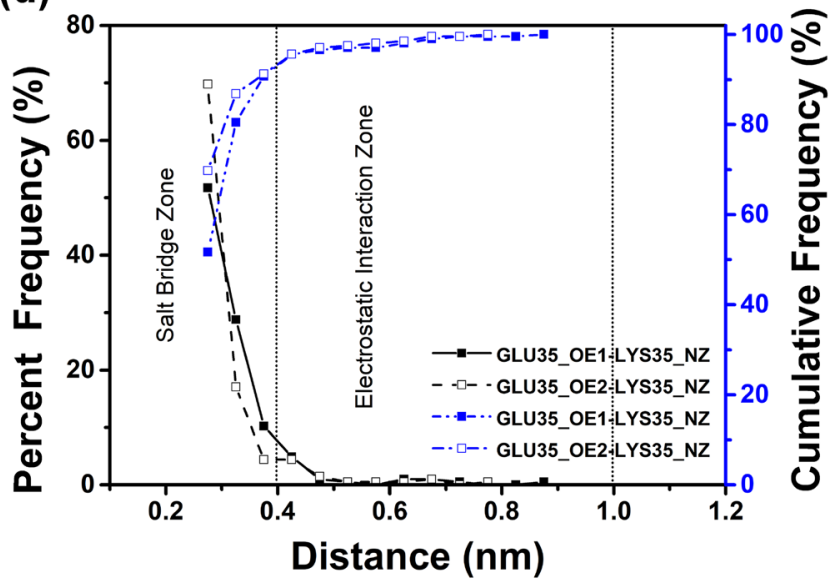

complex; d LYS35 (NZ) and GLU25 (OE1 and OE2) in ACE2-LL37 complex. Here, the distance less than $0.4 \mathrm{~nm}$ belongs to the salt bridge zone, and the distance from $0.4 \mathrm{~nm}$ to $1.0 \mathrm{~nm}$ belongs to the electrostatic interaction zone 
electron microscopy. Hence, inhibiting this residue could help prevent the spread of COVID-19 infections. Indeed, strong foundations are needed to be built to support this hypothesis based on intensive biological experiments and clinical trials.

Similarly, in the ACE2-RBD2 complex, ASP30 (OD1 and OD2) of ACE2 and LYS417 (NZ1) of RBD2 could simultaneously form H-bonds and salt bridges. However, the distance distribution percentage in the salt bridge zone $(<0.4 \mathrm{~nm})$ was $36.94 \%$, and the ratio in the $\mathrm{H}$-bond zone $(<0.35 \mathrm{~nm})$ was $31.76 \%$. Thus, this interaction was sometimes H-bond and salt bridge, and sometimes an electrostatic interaction. Therefore, LYS417 is also a vital amino acid for human-to-human transmission of the SARS-CoV-2 virus.

Additionally, we found that the positive-charged HIS34 of ACE2 and the negative-charged GLU21 of HP5 could form 1-2 strong salt bridges when their distance was less than $0.4 \mathrm{~nm}$ at some intervals. Therefore, we also investigated the distance distribution of HIS34 (NE2) and GLU21 (OE1 and OE2). However, the percentage of the distance distribution in the salt bridge zone was only $5 \%$. And those in the electrostatic interaction zone were up to $94 \%$, which indicated a typical favorable electrostatic interaction.

Interestingly, the distance distribution percentage of GLU35 (OE1 and OE2) of ACE2 and LYS25 (NZ) of LL37 in the salt bridge zone was up to $91.22 \%$. This percentage is 18-fold higher than the HIS34 (NE2) and GLU21 (OE1 and OE2) in the ACE2-HP5 complex, clearly showing its favorability for protein-peptide interfacial binding.

The above results better explain why RBD1 binds strongly to ACE2 than RBD2, and LL37 strongly binds to ACE2 than HD5. In fact, our previous study based on the experimental analysis of LL37 has verified its anti-SARSCoV-2 effect [27].

Generally, molecular simulations can explore the mechanism of intermolecular communications to discover the structural potential and characteristics. Based on such information obtained, one can scan and model drugs at the molecular scale. Though the hypothetical research is still quite distant from the experimental measurement, it appears that experiments can only unveil the overall phenomenon knowing the fact that these studies are laborious and expensive. Several studies on drugs against the SARS-CoV-2 demonstrate that combining computational and in vitro/in vivo assays would yield better results.

\section{Conclusion}

In the current study, we examined the host-guest communication between the human ACE2 and RBD1, RBD2, HP5, and LL37. The results revealed that RBD1 strongly binds to the ACE2 than RBD2. Both the HD5 and LL37 can attach to the human ACE2 and form their stable complexes. Furthermore, HD5 has a potential inhibitory effect on the SARS-CoV-2 and SARS-CoV. The LL37 should have a more substantial inhibitory effect on them as compared to the HD5. Therefore, we anticipate that HD5 and LL37 could be potential drugs for the prevention and treatment of CoVID-19 infection.

Supplementary Information The online version contains supplementary material available at https://doi.org/10.1007/s12539-021-00462-3.

Acknowledgements We owe our gratitude to Jingfei Qiu, Shaomeng Cao and Xuhui Shao from AI Research Center of Peng Cheng Laboratory, Shenzhen, Guangdong province for their enormous technological support in computing resources and technology.

Author Contributions DL supervised research. JQ and TS performed the molecular dynamic simulation. DL and AM wrote the paper. PC designed all figures.

Funding This work was supported by the grants from the Key Research Area Grant 2016YFA0501703 of the Ministry of Science and Technology of China, the National Natural Science Foundation of China (Contract No. 61832019, 61503244), the Science and Technology Commission of Shanghai Municipality (Grant: 19430750600), the Natural Science Foundation of Henan Province (162300410060), as well as SJTU JiRLMDS Joint Research Fund and Joint Research Funds for Medical and Engineering and Scientific Research at Shanghai Jiao Tong University (YG2017ZD14, ZH2018QNA41, YG2019GD01, YG2019ZDA12). Partially Supported by Open Funding Project of State Key Laboratory of Microbial Metabolism (MMLKF21-11) and Shanghai cryogenic biomedical technology professional service platform (18DZ2295700).

Data Availability Table S1 Solvent accessible interface areas of four equilibrated complex structures; Table S2 H-bond interaction between ACE2 and HP5; Table S3 Electrostatic interaction between ACE2 and HP5; Table S4 Hydrophobic interaction between ACE2 and HP5; Table S5 H-bond interaction between ACE2 and LL-37; Table S6 Salt bridges between ACE2 and LL-37; Table S7 Electrostatic interaction between ACE2 and LL-37; Table S8 Hydrophobic interactions.

Code Availability Not applicable.

\section{Declarations}

Conflict of Interest We have no competing financial interests to declare.

Ethical Approval Not applicable.

Consent to Participate Not applicable.

Consent for Publication Not applicable.

\section{References}

1. Yan R et al (2020) Structural basis for the recognition of SARSCoV-2 by full-length human ACE2. Science 367(6485):14441448. https://doi.org/10.1126/science.abb2762 
2. Wan Y et al (2020) Receptor recognition by the novel coronavirus from Wuhan: an analysis based on decade-long structural studies of SARS coronavirus. J Virol. https://doi.org/10.1128/JVI. 00127-20

3. Vashisht R, Duggal A (2020) Respiratory failure in patients infected with SARS-CoV-2. Cleve Clin J Med. https://doi.org/ 10.3949/ccjm.87a.ccc025

4. Turtle L (2020) Respiratory failure alone does not suggest central nervous system invasion by SARS-CoV-2. J Med Virol 92(7):705706. https://doi.org/10.1002/jmv.25828

5. Agostoni $\mathrm{P}$ et al (2020) Cardiac patient care during a pandemic: how to reorganise a heart failure unit at the time of COVID-19. Eur J Prev Cardiol 27(11):1127-1132. https://doi.org/10.1177/ 2047487320925632

6. Maisch B (2020) SARS-CoV-2 as potential cause of cardiac inflammation and heart failure. Is it the virus, hyperinflammation, or MODS? Herz 45(4):321-322. https://doi.org/10.1007/ s00059-020-04925-z

7. Palumbo D, Guazzarotti G, De Cobelli F (2020) Spontaneous major hemorrhage in COVID-19 patients: another brick in the wall of SARS-CoV-2-associated coagulation disorders? J Vasc Interv Radiol. https://doi.org/10.1016/j.jvir.2020.06.010

8. Qiu H et al (2020) Acute on chronic liver failure from novel severe acute respiratory syndrome coronavirus 2 (SARS-CoV-2). Liver Int 40(7):1590-1593. https://doi.org/10.1111/liv.14506

9. Sardu C et al (2020) Hypertension, thrombosis, kidney failure, and diabetes: is COVID-19 an endothelial disease? A comprehensive evaluation of clinical and basic evidence. J Clin Med. https://doi. org $/ 10.3390 /$ jcm 9051417

10. Ou X et al (2020) Characterization of spike glycoprotein of SARS-CoV-2 on virus entry and its immune cross-reactivity with SARS-CoV. Nat Commun 11(1):1620. https://doi.org/10.1038/ s41467-020-15562-9

11. Walls AC et al (2020) Structure, function, and antigenicity of the SARS-CoV-2 spike glycoprotein. Cell 181(2):281-292.e6. https:// doi.org/10.1016/j.cell.2020.02.058

12. Wrapp D et al (2020) Cryo-EM structure of the 2019-nCoV spike in the prefusion conformation. Science 367(6483):1260-1263. https://doi.org/10.1126/science.abb2507

13. Wang $\mathbf{J}$ et al (2020) Molecular simulation of SARS-CoV-2 spike protein binding to pangolin ACE2 or human ACE2 natural variants reveals altered susceptibility to infection. J Gen Virol. https:// doi.org/10.1099/jgv.0.001452

14. Dugan AS et al (2008) Human alpha-defensins inhibit BK virus infection by aggregating virions and blocking binding to host cells. J Biol Chem 283(45):31125-31132. https://doi.org/10.1074/ jbc.M805902200

15. Durr UH, Sudheendra US, Ramamoorthy A (2006) LL-37, the only human member of the cathelicidin family of antimicrobial peptides. Biochim Biophys Acta 1758(9):1408-1425. https://doi. org/10.1016/j.bbamem.2006.03.030

16. Heilborn JD et al (2005) Antimicrobial protein hCAP18/LL-37 is highly expressed in breast cancer and is a putative growth factor for epithelial cells. Int J Cancer 114(5):713-719. https://doi.org/ 10.1002/ijc.20795

17. Mendez-Samperio P (2010) The human cathelicidin hCAP18/ LL-37: a multifunctional peptide involved in mycobacterial infections. Peptides 31(9):1791-1798. https://doi.org/10.1016/j.pepti des.2010.06.016

18. Kai-Larsen Y, Agerberth B (2008) The role of the multifunctional peptide LL-37 in host defense. Front Biosci 13:3760-3767. https://doi.org/10.2741/2964

19. Gordon YJ et al (2005) Human cathelicidin (LL-37), a multifunctional peptide, is expressed by ocular surface epithelia and has potent antibacterial and antiviral activity. Curr Eye Res 30(5):385-394. https://doi.org/10.1080/02713680590934111
20. Bergman $P$ et al (2007) The antimicrobial peptide LL-37 inhibits HIV-1 replication. Curr HIV Res 5(4):410-415. https://doi. org/10.2174/157016207781023947

21. Bourgade $\mathrm{K}$ et al (2015) beta-Amyloid peptides display protective activity against the human Alzheimer's disease-associated herpes simplex virus-1. Biogerontology 16(1):85-98. https:// doi.org/10.1007/s10522-014-9538-8

22. Wang G et al (2014) High-quality 3D structures shine light on antibacterial, anti-biofilm and antiviral activities of human cathelicidin LL-37 and its fragments. Biochim Biophys Acta 1838(9):2160-2172. https://doi.org/10.1016/j.bbamem.2014. 01.016

23. Harcourt JL et al (2016) Human cathelicidin, LL-37, inhibits respiratory syncytial virus infection in polarized airway epithelial cells. BMC Res Notes 9:11. https://doi.org/10.1186/ s13104-015-1836-y

24. Diamond $\mathrm{G}$ et al (2021) Potent antiviral activity against HSV-1 and SARS-CoV-2 by antimicrobial peptoids. Pharmaceuticals (Basel). https://doi.org/10.3390/ph14040304

25. Roth A et al (2020) LL-37 fights SARS-CoV-2: the vitamin D-inducible peptide LL-37 inhibits binding of SARS-CoV-2 spike protein to its cellular receptor angiotensin converting enzyme 2 in vitro. bioRxiv. https://doi.org/10.1101/2020.12. 02.408153

26. Hartshorn KL (2020) Innate immunity and influenza A virus pathogenesis: lessons for COVID-19. Front Cell Infect Microbiol 10:563850. https://doi.org/10.3389/fcimb.2020.563850

27. Wang $\mathrm{C}$ et al (2021) Human cathelicidin inhibits SARS-CoV-2 infection: killing two birds with one stone. ACS Infect Dis. https:// doi.org/10.1021/acsinfecdis.1c00096

28. Wang G (2008) Structures of human host defense cathelicidin LL-37 and its smallest antimicrobial peptide KR-12 in lipid micelles. J Biol Chem 283(47):32637-32643. https://doi.org/10. 1074/jbc.M805533200

29. Song $\mathrm{W}$ et al (2018) Cryo-EM structure of the SARS coronavirus spike glycoprotein in complex with its host cell receptor ACE2. PLoS Pathog 14(8):e1007236. https://doi.org/10.1371/journal. ppat. 1007236

30. Wang $\mathrm{C}$ et al (2020) Human intestinal defensin 5 inhibits SARSCoV-2 invasion by cloaking ACE2. Gastroenterology. https://doi. org/10.1053/j.gastro.2020.05.015

31. Pierce BG et al (2014) ZDOCK server: interactive docking prediction of protein-protein complexes and symmetric multimers. Bioinformatics 30(12):1771-1773. https://doi.org/10.1093/bioin formatics/btu097

32. Rakhshani H, Dehghanian E, Rahati A (2019) Enhanced GROMACS: toward a better numerical simulation framework. J Mol Model 25(12):355. https://doi.org/10.1007/ s00894-019-4232-z

33. Pang YP (2016) FF12MC: a revised AMBER forcefield and new protein simulation protocol. Proteins 84(10):1490-1516. https:// doi.org/10.1002/prot.25094

34. Sukhwal A, Sowdhamini R (2013) Oligomerisation status and evolutionary conservation of interfaces of protein structural domain superfamilies. Mol Biosyst 9(7):1652-1661. https://doi. org/10.1039/c3mb25484d

35. Aldeghi M et al (2017) Statistical analysis on the performance of molecular mechanics Poisson-Boltzmann surface area versus absolute binding free energy calculations: bromodomains as a case study. J Chem Inf Model 57(9):2203-2221. https://doi.org/ 10.1021/acs.jcim.7b00347

36. Kumari R et al (2014) g_mmpbsa-a GROMACS tool for high-throughput MM-PBSA calculations. J Chem Inf Model 54(7):1951-1962. https://doi.org/10.1021/ci500020m

37. Iida S, Nakamura H, Higo J (2016) Enhanced conformational sampling to visualize a free-energy landscape of protein complex 
formation. Biochem J 473(12):1651-1662. https://doi.org/10. 1042/BCJ20160053

38. Li F et al (2005) Structure of SARS coronavirus spike receptor-binding domain complexed with receptor. Science 309(5742):1864-1868. https://doi.org/10.1126/science.1116480

39. Elbe S, Buckland-Merrett G (2017) Data, disease and diplomacy: GISAID's innovative contribution to global health. Glob Chall 1(1):33-46. https://doi.org/10.1002/gch2.1018
40. Veeramachaneni GK et al (2020) Structural and simulation analysis of hotspot residues interactions of SARS-CoV 2 with human ACE2 receptor. J Biomol Struct Dyn. https://doi.org/10.1080/ 07391102.2020 .1773318

\section{Authors and Affiliations}

\section{Daixi Li ${ }^{1}\left(\mathbb{0} \cdot\right.$ Peiqin Chen ${ }^{1} \cdot$ Ting Shi $^{2} \cdot$ Aamir Mehmood $^{2} \cdot$ Jingfei Qiu $^{3}$}

1 Institute of Biothermal Science and Technology, University of Shanghai for Science and Technology, Shanghai 200093, China

2 State Key Laboratory of Microbial Metabolism, Joint International Research Laboratory of Metabolic and Developmental Sciences, School of Life Sciences and Biotechnology, Shanghai Jiao Tong University, Shanghai 200240, People's Republic of China

3 AI Research Center, Peng Cheng Laboratory, Shenzhen, Guangdong 518055, People's Republic of China 